\title{
Spatiotemporal nonlinearity in resting-state fMRI of the human brain
}

\author{
Xiaoping Xie, ${ }^{\mathrm{a}, *}$ Zhitong Cao, ${ }^{\mathrm{a}}$ and Xuchu Weng ${ }^{\mathrm{b}}$ \\ ${ }^{a}$ Physics Department, Zhejiang University, Hangzhou, China \\ ${ }^{\mathrm{b}}$ Psychology Research Institute, Chinese Academy of Sciences, Beijing, China
}

Received 30 April 2007; revised 4 January 2008; accepted 11 January 2008

Available online 17 January 2008

\begin{abstract}
In this work, the spatiotemporal nonlinearity in resting-state fMRI data of the human brain was detected by nonlinear dynamics methods. Nine human subjects during resting state were imaged using single-shot gradient echo planar imaging on a 1.5T scanner. Eigenvalue spectra for the covariance matrix, correlation dimensions and Spatiotemporal Lyapunov Exponents were calculated to detect the spatiotemporal nonlinearity in resting-state fMRI data. By simulating, adjusting, and comparing the eigenvalue spectra of pure correlated noise with the corresponding real fMRI data, the intrinsic dimensionality was estimated. The intrinsic dimensionality was used to extract the first few principal components from the real fMRI data using Principal Component Analysis, which will preserve the correct phase dynamics, while reducing both computational load and noise level of the data. Then the phase-space was reconstructed using the time-delay embedding method for their principal components and the correlation dimension was estimated by the Grassberger-Procaccia algorithm of multiple variable series. The Spatiotemporal Lyapunov Exponents were calculated by using the method based on coupled map lattices. Through nonlinearity testing, there are significant differences of correlation dimensions and Spatiotemporal Lyapunov Exponents between fMRI data and their surrogate data. The fractal dimension and the positive Spatiotemporal Lyapunov Exponents characterize the spatiotemporal nonlinear dynamics property of resting-state fMRI data. Therefore, the results suggest that fluctuations presented in resting state may be an inherent model of basal neural activation of human brain, cannot be fully attributed to noise.
\end{abstract}

(C) 2008 Elsevier Inc. All rights reserved.

Keywords: Spatiotemporal nonlinearity; Correlation dimension; Spatiotemporal Lyapunov Exponents; Intrinsic dimensionality; Principal Component Analysis; fMRI; Resting-state; Human brain

\section{Introduction}

Functional magnetic resonance imaging (fMRI) has emerged as a useful and noninvasive technique for the study of structure-function

\footnotetext{
* Corresponding author.

E-mail address: iap@zju.edu.cn (X. Xie).

Available online on ScienceDirect (www.sciencedirect.com).
}

relationship in the human brain. Using magnetic resonance techniques, researchers have found that it is possible to indirectly detect changes in blood-oxygenation levels that are a result of neuronal activation. In the past decade interest in this novel research field increased rapidly. Most of the work concentrated on the detection or the estimation of brain regions involved in specific cognitive or sensor-motor tasks.

The complex behavior of the hemodynamic response is a global phenomenon and the reconstruction of the dynamics recorded in fMRI data should make use of the vast amount of spatial information acquired (Laird et al., 2002). Spatiotemporal electroencephalography (EEG) and magnetoencephalography (MEG) signal analysis can achieve higher performance by combining spatial and temporal approaches (Lee and Kim, 2006; Uhl et al., 1998; Pezard et al., 1996). Compared with EEGs of low spatial resolution, fMRI data offer millimeter spatial resolution (1 to $4 \mathrm{~mm}$ ) with temporal resolutions of the order of seconds. It can offer more spatial information than EEG/MEG. Hence, spatiotemporal analysis by fMRI will produce an important analytic tool for brain research (McIntosh et al., 2004).

Conscious rest has been widely used as a baseline condition in neuroimaging experiments such as positron emission tomography (PET) and functional magnetic resonance imaging (Gusnard and Raichle, 2001). In most cases, rest state is defined as a state that differs from the active state both in terms of conditions (open/ closed eyes, absence/presence of a stimulus input) and instructions given to the subject (Wicker et al., 2003). A rest state can therefore be used in a wide variety of experiments. However, it is an illdefined mental state because it may vary both from one subject to another and within the same subject (Luca et al., 2006; Wicker et al., 2003).

Extracting information from resting-state is a challenging work, because the signals of interest are contaminated by physiological noise, such as breathing, cardiac activity (Thirion et al., 2006) and scan noise. Many methods are introduced to analyze resting-state fMRI data, such as Fourier Transformation (Cordes et al., 2001), Correlation Analysis (Hampson et al., 2002; Cordes et al., 2001; Lowe et al., 1998), Principal Component Analysis (Worsley et al., 2005; Zuendorf et al., 2003), and Independent Component Analysis (Luca et al., 2006; Bartels and Zeki, 2005). However, 
the majority of methods developed for resting-state fMRI data analysis so far are linear in nature.

Recently, the analysis of human EEGs with methods based on nonlinear dynamics and chaos theory has become increasingly popular (Lehnertz and Elger, 1998; Pezard et al., 1996). One of the most important contributions of nonlinear dynamics to the general view of the physical world is the message that irregular and seemingly unpredictable behaviors do not necessarily have to be attributed to some random external inputs to the systems, but on the contrary can be the result of completely deterministic dynamical systems (nonlinearity is a necessary). This new paradigm offers a possible new way to the analysis of many irregular time series, which have been regarded only as stochastic signals before the breakthrough of nonlinear dynamics (Galka, 2000). The application of nonlinear dynamics analysis tools to characterize time series may provide a more complete description of EEG recordings (Stam, 2005; Lee et al., 2001). It has been regarded as an important advance in understanding the underlying mechanism of brain electrical activities. It has been realized that if chaos could be demonstrated in a natural system, such as the human brain, it might provide a much simpler explanation for the occurrence of complex behavior than usual stochastic models (Lee et al., 2001). The presence of nonlinear or deterministic chaotic behavior in various physiological and pathological states has been postulated, but also disputed (Freeman, 2000; Stam et al., 1995). Therefore, detection of nonlinearity is important and should be the first step before any nonlinear analysis.

Even though there is an effort to study nonlinear dynamics of brain activities using EEG, very little research has been done in applying methods of nonlinear dynamics to fMRI data, particularly during resting state of the human brain. Recently, the nonlinear dynamics analysis of fMRI data of the human brain has begun to attract many researchers. An extension of the delta-epsilon approach is applied to fMRI data to evaluate whether a time course of a candidate voxel provides additional information concerning the time evolution of reference voxel time series (LaConte et al., 2004). The nonlinearity arising from the finite dimensional dynamics are then characterized using patterns of singularities in the complex plane. A finite embedding dimension is a measure of the determinism of the system, which can be quantified using information theoretic measures like Lempel-Ziv complexity (Deshpande et al., 2006). Using spatial embedding of fMRI data, local spatiotemporal chaos in baseline (Deshpande et al., 2005) has been reported. However, most works on nonlinear analysis to fMRI data are taken voxel by voxel based on single time series (Gautanma et al., 2003), as is traditionally done in the nonlinear signal processing literature. On the other hand, though recent studies (Vazquez and Noll, 1998; Birn et al., 2001; Bandettini et al., 2002; Pfeuffer et al., 2003; Huettel, 2004) indicate the nonlinear nature of fMRI response to some stimulation and many nonlinear models between stimulations and their fMRI responses are established (Wager et al., 2005; Harrison et al., 2003; Friston et al., 2000; Buxton et al., 1998), these results are obtained by means of stimulations and their fMRI responses. However, in resting-state of the human brain, there are no significant stimulations. Hence, it is difficult to detect the nonlinearity by way of stimulations and their fMRI responses.

In this work, the nonlinearity in resting-state fMRI signals of the human brain was detected using two methods which are usually used to characterize the essence of a nonlinear dynamical system. One is the correlation dimension analysis which analyzes quantitatively the nonlinear fractal property of fMRI data of the human brain. Another is the Spatiotemporal Lyapunov Exponent
(STLE) analysis which characterizes the nonlinear chaotic property of fMRI data of the human brain.

Because various dynamical quantities of the reconstructed set or phase-space are the same as those of the underlying attractor (Kantz and Schreiber, 1997), provided that embedding dimension is suitably large, an appropriate phase-space reconstruction has to be carried out before estimating the correlation dimension of fMRI data using Grassberger-Procaccia algorithm. The so-called "appropriate" is that the reconstructed set not only comprises essential information and noise-free, but also has appropriate calculation quantity and as less redundancy as possible. So knowing the essential number of signal components is a key step for popular fMRI data postprocessing.

In order to estimate accurately the number of essential signals for the resting-state fMRI data of the human brain, i.e. the intrinsic dimension, a method based on an autoregressive noise model of order 1, AR(1) noise model, was used to estimate the intrinsic dimensionality of fMRI data and cubic spline interpolation was introduced to the estimate of AR(1) coefficient $\phi$. According to the estimated intrinsic dimensionality, the principal components of fMRI data were extracted by Principal Component Analysis (PCA) method. The phase-space was reconstructed using the time-delay embedding method for their principal components. In the reconstructed phase-space, the correlation dimension of spatiotemporal series was estimated using Grassberger-Procaccia algorithm. The important result of fractal correlation dimension characterizes quantitatively the nonlinear fractal property of resting-state fMRI data of human brain.

On the other hand, the global coupled STLE, which is based on coupled map lattices (CML), was introduced to analyze spatiotemporal series of fMRI data in resting-state of human brain. The result of positive STLE, which characterizes the chaotic nonlinear dynamical property, was also obtained.

In the end, two kinds of surrogate data generated from raw fMRI data were introduced to test the nonlinearity in resting-state fMRI data of human brain.

\section{Material and methods}

\section{Correlation dimension analysis}

\section{Correlation dimension}

The correlation dimension is a method aiming at practical applications where the geometrical object has to be reconstructed from a finite sample of data points which mostly contain some errors as well. The Grassberger-Procaccia algorithm (Kantz and Schreiber, 1997; Hegger and Kantz, 1999), which is the most popular method to estimate the correlation dimension, is based on an appropriate phase-space reconstruction. One of the most popular methods for phase-space reconstruction is time-delay embedding (Bianciardi et al., 2007; Perc, 2005; Cellucci et al., 2003; Hegger and Kantz, 1999). Given a scalar time series $x(t)$, a sequence of vectors $y(t)=$ $(x(t), x(t+\tau), \cdots, x(t+[m-1] \tau))$ is formed, where $m$ is embedding dimension and $\tau$ is the delay time. Thus, a phase space of $m$ dimensions is reconstructed by this sequence of vectors. Under quite general circumstances the attractor formed by delay embedding is equivalent to the attractor in unknown space in which the underlying dynamical system is living if the embedding dimension $m$ of the delay coordinate space is sufficiently large.

Meanwhile it is assumed that there are only a finite number of points which are generated by a dynamic system. After phase space 
reconstruction, $M_{\mathrm{y}}$ vectors (denoted by $y_{\mathrm{i}}, i=0,1, \ldots, M_{\mathrm{y}}-1$ ) are reformed. The next step of correlation dimension estimation is to calculate the correlation integral (such an approximation of correlation integral will be termed correlation sum). Due to the existence of temporal correlations in the fMRI data, Theiler correlation sum (Small, 2005) is calculated. That is, those pairs of points which are close in time are excluded. The correlation sum is started after a time (W) which is determined by the average correlation time, i.e. the time when the autocorrelation function has decayed to $1 / \mathrm{e}$. Since there are only a finite number of points, the Theiler correlation integral is approximated by

$$
C(\varepsilon)=\frac{2}{\left(M_{y}-W\right)\left(M_{y}-W-1\right)} \sum_{i} \sum_{j>i+W} \Theta\left(\varepsilon-\left\|y_{i}-y_{j}\right\|\right)
$$

where $\Theta$ is the Heaviside step function, $\Theta(x)=0$ if $x \leq 0$ and $\Theta(x)=1$ if $x>0$. The correlation sum just counts the pairs $\left(y_{\mathrm{i}}, y_{\mathrm{j}}\right)$ whose distance (denoted by $\|\bullet\|$ ) in phase space is smaller than $\varepsilon$. In the limit of an infinite of the data $\left(M_{\mathrm{y}} \rightarrow \infty\right)$ and for a small given $\varepsilon$, a power law (Kantz and Schreiber, 1997) will be expected to scale $C(\varepsilon)$.

$C(\varepsilon) \propto \varepsilon^{d_{2}}$

Thus, the correlation dimension can be defined by

$d_{2}=\lim _{\varepsilon \rightarrow 0} \frac{\log C(\varepsilon)}{\log \varepsilon}$

In practice, a typical correlation integral plot will contain a "scaling region" over which the slope of $\log C(\varepsilon)$ remains relatively constant (Galka, 2000). A common way to examine the slope in the scaling region is to numerically differentiate (or fit a line to) the plot of $\log C(\varepsilon)$ against $\log \varepsilon$. This ought to produce a function which is constant over the scaling region, and its value on this region should be the correlation dimension.

Multivariate data, such as spatiotemporal series, can be assumed to be either independent realizations of the same observable in a given system or different observables of a single attractor measured simultaneously (Dhamala et al., 2002). Within the general framework, multivariate data can be treated in very much the same way as scalar time series. With embedding each time series separately, the Theiler correlation sum algorithm can be easily extended to multivariate data. Thus, for multivariate data which are composed of $K$ numbers of time series, the multivariate Theiler correlation sum can be written as

$C_{M_{T}}(\varepsilon)=\frac{2}{\left(M_{T}-W\right)\left(M_{T}-W-1\right)} \sum_{j=1}^{M_{T}} \sum_{i=j+W+1}^{M_{T}} \Theta\left(\varepsilon-\left\|Y_{i}-Y_{j}\right\|\right)$

where both $Y_{\mathrm{i}}$ and $Y_{\mathrm{j}}$ belong to the set of all the delayed vectors $Y=\left\{\left\{x_{i}^{1}\right\}_{i=1}^{M_{1}},\left\{x_{i}^{2}\right\}_{i=1}^{M_{2}}, \ldots,\left\{x_{i}^{K}\right\}_{i=1}^{M_{K}}\right\}$, if $x_{i}^{(1,2, \ldots, K)}$ denotes the delay vectors constructed from individual time series and $M_{1}, M_{2}, \cdots, M_{\mathrm{K}}$ are the numbers of delayed vectors in the reconstructed space for $K$ time series $\left(M_{\mathrm{T}}=M_{1}+M_{2}+\cdots+M_{\mathrm{K}}\right)$. As in scalar time series, the correlation sum of multivariate data also counts the probability of the pairs $\left(Y_{\mathrm{i}}, Y_{\mathrm{j}}\right)$ whose distance in the reconstructed phase space is smaller than $\varepsilon$. The correlation dimension $d_{2}$ is then determined from the "scaling region" plateaus (corresponding to different embedding dimension $m$ ) in the plot of $\operatorname{dlog}_{2}\left[C_{M_{T}}(m, \varepsilon)\right] / \operatorname{dlog}_{2}(\varepsilon)$ against $\log _{2}(\varepsilon)$ by the same procedure as scalar time series. Within this "scaling region", the correlation sum is well described by a power law. Although there lacks a fundamental mathematical understanding, the validity (Dhamala et al., 2002) of the phase-space reconstruction from multiple series has been checked with mathematical models and multiple channel EEG data.

\section{Principal Component Analysis}

Although the input dimensionality of fMRI data may be quite high (e.g., 600 images for $64 \times 64 \times 5$ voxels), the meaningful structure of these data has many fewer independent degrees of freedom. On the other hand, direct estimation of the correlation dimension for fMRI data will become very difficult because of the massive calculation quantity. Therefore, dimensionality reduction, namely finding meaningful low-dimensional structures hidden in their high-dimensional observations, is a key step to deal with large volumes of high-dimensional data.

Principal Component Analysis (PCA) is a common method to reduce the dimensionality and to extract important modes of activity from massive data such as fMRI data of the human brain (Cordes and Nandy, 2006). Implementation of PCA (Kantz and Schreiber, 1997; Hegger and Kantz, 1999) amounts to a search for the direction of maximum variance in the data, followed by an orthogonal projection of the data onto a subspace spanned by direction vectors with highest variance. Algorithmically, PCA calculate an eigenvalue decomposition of the sample covariance matrix $R$ of $M \times N$ real matrix $\boldsymbol{X}$ (for fMRI data, $M$ is the number of time points and $N$ is the number of voxels). Since the covariance matrix $R$ is a real symmetric matrix, its eigenvalues are real and its eigenvectors are orthogonal.

PCA is also easily implemented by singular value decomposition of the data matrix (Dhamala et al., 2002; Thirion et al., 2006; Reidl et al., 2007). For a $M \times N$ real matrix $\boldsymbol{X}$, singular value decomposition is to factorize the matrix $\boldsymbol{X}$ into the product of matrices $U, S, V$ such as $\boldsymbol{X}=U S V^{\mathrm{T}}$, where both $U$ and $V$ are orthogonal matrices of sizes $M \times M$ and $N \times N$ respectively, and $S$ is a $M \times N$ diagonal matrix with the singular values $\sigma_{1} \geq \cdots \geq \sigma_{\min (M, N)} \geq 0$.

PCA is essentially a linear method. The reconstruction itself is just a linear combination. It has been indicated (Dhamala et al., 2002) that PCA can extract qualitatively nonlinear dynamical features from experimental time series. Hence, taking the first few principal components will preserve the correct phase dynamics in the reconstructed space while it reduces the noise level of the data. At the same time, due to reducing the dimensions of the data, the calculation quantity is decreased significantly.

\section{Intrinsic dimensionality estimation}

In order to preserve the correct phase dynamics in reconstructed space, a key step to use the popular tools like PCA for fMRI data post-processing is to determine the number of essential components, i.e. the intrinsic dimensionality of fMRI data. A method (Cordes and Nandy, 2006) based on an autoregressive noise model of order 1 is used to detect the number of signal components in fMRI data.

Typical fMRI data are composed of signals from specific cognitive and other physiological processes contaminated by noise, which arises from several different sources such as thermal noise and scanner noise. Hence, fMRI data can be described by the noise linear mixing model

$x_{\mathrm{i}}=\boldsymbol{A} s_{\mathrm{i}}+\eta_{\mathrm{i}}, \quad i=1, \ldots, N$

where $i$ represents the voxel, $N$ is the number of voxels, $x_{\mathrm{i}}$ is the observed time course with $M$ time points at voxel $i, \boldsymbol{A}$ is the $M \times p$ 
dimensional mixing matrix, $s_{\mathrm{i}}$ is the signal vector with $p$ components at voxel $i$, and $\eta_{\mathrm{i}}$ is the noise vector which follows a multivariate Gaussian distribution with zero mean and covariance matrix $\sum . \boldsymbol{X}=\left[x_{1}, \ldots, x_{\mathrm{N}}\right]$ is the observation matrix. Though the number of "true" biological components of the signal may be less than $M$ (i.e. $p<M$ ), due to the presence of noise and $N$ being larger than $M, X$ is always a matrix of full rank $M$. This means that the dimensionality of full data is always larger than the number of "true" components.

It is shown (Maxim et al., 2005) that noise in a voxel time series of fMRI data is not uncorrelated but has an autoregressive structure, even when the data have been acquired with the subject "at rest", and the autoregressive linear time invariant models (AR) are usually used to model the fMRI noise. Although the variability of autocorrelation among voxels suggests that it might be suitable for adapting the order of AR process to each individual time series, it is proved (Cordes and Nandy, 2006) that modeling the noise by an $\mathrm{AR}(1)$ process seems to work well and leads to consistent intrinsic dimensionality estimates for simulated data, water phantom data and also preprocessed real data (having corrected motion artifacts and detrended signal drifts).

Correlated Gaussian noise $\eta(t)$, satisfying an $\mathrm{AR}(1)$ noise model, is defined by:

$\eta(t)=\phi \eta(t-1)+\varepsilon(t)$

where $\phi$ is the $\operatorname{AR}(1)$ coefficient and $\varepsilon(t)$ is a random variable with Gaussian distribution $N\left(0, \sigma^{2}\right)$.

Simulation (Cordes and Nandy, 2006) shows that within the scope of $\phi \in[0,0.3], \lambda(k)$, the $k$-th eigenvalue of the sample covariance matrix $R$, can be approximated by an exponential function

$\lambda(k)=a \mathrm{e}^{-b k}, k \in[1, M]$

where $a(\phi)$ and $b(\phi)$ are the coefficients. Small deviations from the exponential behavior only occur for very small $k(k<10)$ or very large $k(k>M-10)$. Cordes and Nandy also indicate that both the coefficients $a(\phi)$ and $b(\phi)$ can also be fitted by exponential functions for the AR(1) coefficient $\phi \in[0,0.3]$ and typical fMRI parameters (the number of voxels $N=20,000$ and the temporal size $M=160)$.

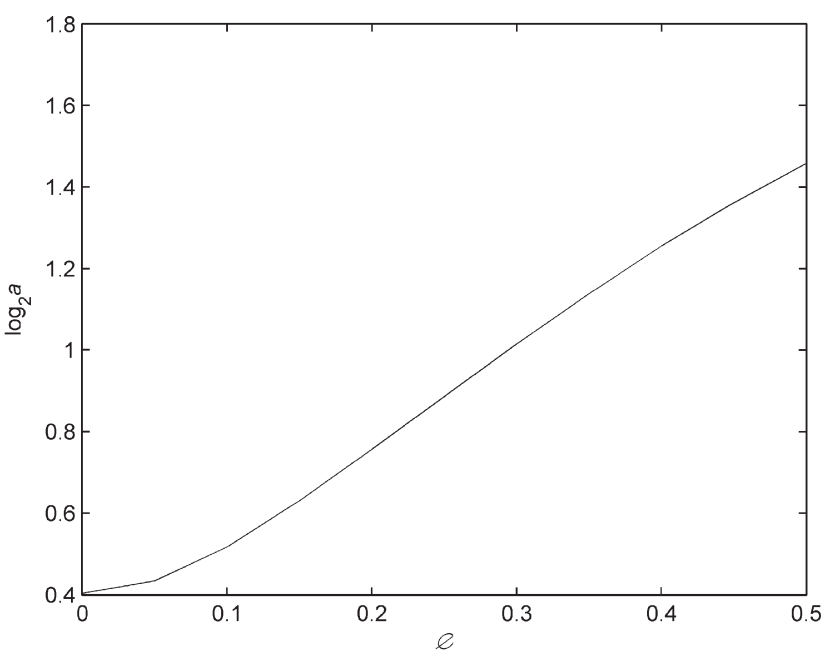

Nevertheless, for small $N$, we find that neither $a(\phi)$ against $\phi$ nor $b(\phi)$ against $\phi$ can be accurately parameterized by an exponential function. As $N=20000$, Fig. 1 suggests that the points of $\log a(\phi)$ against $\phi$ fall near a straight line for $\phi \in[0.05,0.30]$, i.e. the plot of $a(\phi)$ against $\phi$ can be accurately parameterized by an exponential function within this scope of $\phi$. The plot of $b(\phi)$ against $\phi$ is the same. However, as $N=1539$, both the plot of $\log a(\phi)$ against $\phi$ and $\log b(\phi)$ against $\phi$ are nonlinear for $\phi \in[0.05,0.30]$ as shown in Fig. 2. In other words, neither the coefficients of $a(\phi)$ against $\phi$ nor the coefficients of $b(\phi)$ against $\phi$ can be accurately fitted by an exponential function. This implies that the method based on an exponential function cannot obtain an accurate estimate of the AR(1) coefficient $\phi$ on condition that $N$ is small.

Interpolation is usually used to estimate the value of a function between known data points without knowing the actual function. Cubic spline interpolation (Burden and Faires, 2001) is a useful technique to interpolate between known data points due to its stable and smooth characteristics. They are generally well behaved and continuous up to the second order derivative at the data points. In this work, after obtaining the plot of coefficient $b(\phi)$ against $\phi$, we introduced cubic spline interpolation to estimate the $\operatorname{AR}(1)$ coefficient $\phi$ corresponding to the tail eigenvalues of the covariance matrix for fMRI data.

The algorithm to estimate the intrinsic dimensionality is carried out by the following steps:

Step 1. Construct $a(\phi)$ and $b(\phi)$ by simulation data in noise space. The $a(\phi)$ and $b(\phi)$ are constructed by using the simulation data of pure Gaussian noise with $\mathrm{AR}(1)$ covariance structure. The simulation data has the same $M$ and $N$ as the corresponding fMRI data. All $\phi$ coefficients are within the scope of $[0,0.5]$. Their interval is 0.05 . Thus the plot of $a(\phi)$ against $\phi$ and the plot of $b(\phi)$ against $\phi$ are obtained.

Step 2. Determine the proper $\phi_{\gamma}$ corresponding to fMRI data. In order to determine the proper $\phi_{\gamma}$ corresponding to fMRI data, the eigenvalue spectrum of real fMRI data is calculated. The tail eigenvalues of real fMRI data, excluding the very last 10 eigenvalues (due to the presence of colinearities in the data, the last few eigenvalues can be close to zero, and should therefore be excluded from the

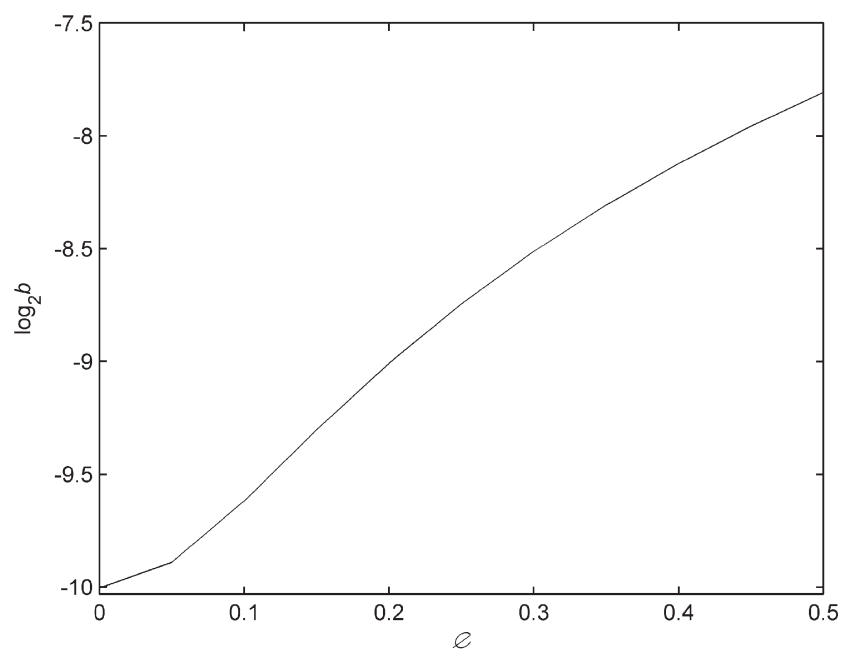

Fig. 1. A typical coefficients $a(\phi)$ against $\phi$ and $b(\phi)$ against $\phi$ for correlated Gaussian noise corresponding to an AR(1) model (600 time dimension, 20,000 samples). $\phi$ is the AR(1) coefficient. 

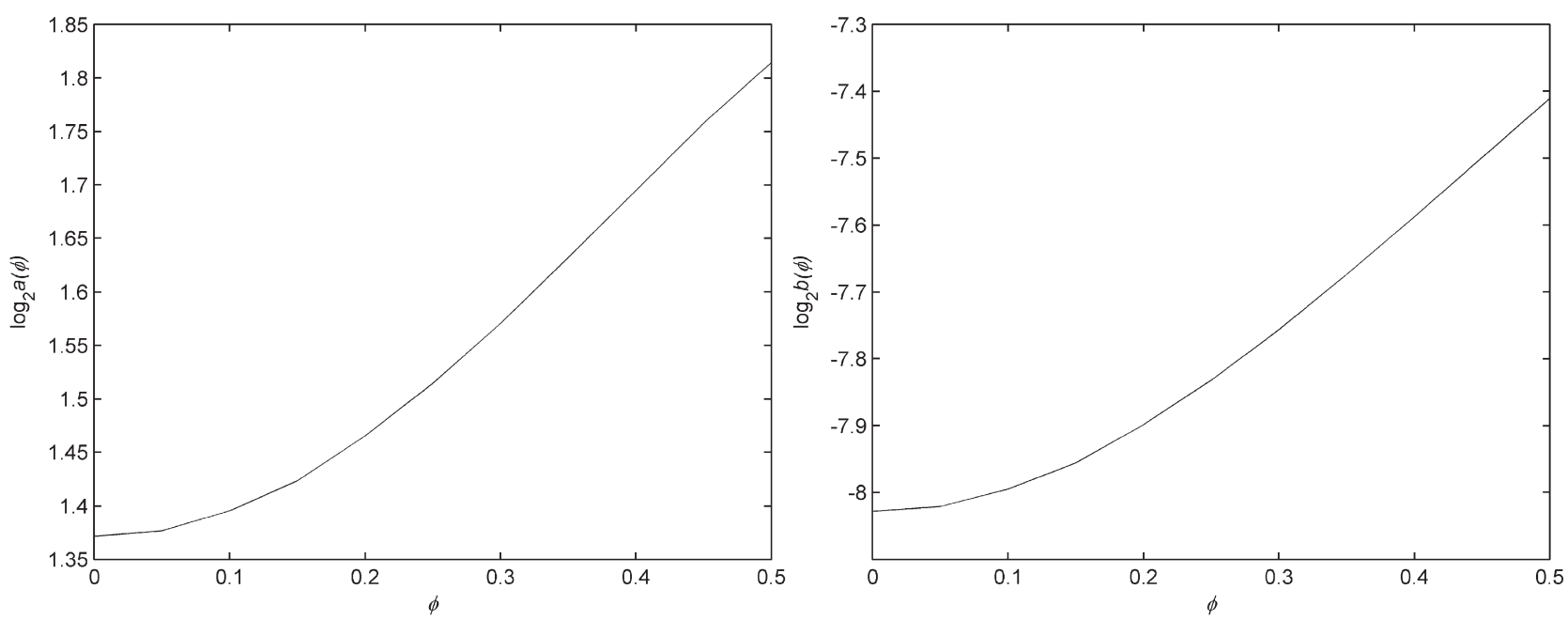

Fig. 2. A plot of coefficients $a(\phi)$ against $\phi$ and $b(\phi)$ against $\phi$ for correlated Gaussian noise corresponding to an AR(1) model. $\phi$ is the AR(1) coefficient and $\phi \in[0,0.5]$. The time dimension is 600 and the number of voxels is 1539 .

purpose of dimensionality estimation), are fitted to $\lambda(k)=$ $a_{\gamma} e^{-b_{\gamma} k}$, and the coefficients $a_{\gamma}$ and $b_{\gamma}$ can be obtained. Assuming that the tail eigenvalues of the covariance matrix of real fMRI data will have no contributions for the signals, the proper $\phi_{\gamma}$ at the point of obtained coefficient $b_{\gamma}$ corresponding to fMRI data, which is also the $\operatorname{AR}(1)$ coefficient, can be estimated by interpolating within the constructed points of $(b(\phi), \phi)$ using cubic spline interpolation.

Step 3. Adjust the eigenvalue spectrum of the simulated AR(1) noise data. With the proper coefficient $\phi_{\gamma}$ estimated from fMRI data in step 2, the simulated AR(1) noise data are generated again using the same $M$ and $N$ as the corresponding fMRI data. Because of the variance normalization of each series, the tail eigenvalues for real fMRI data will always be smaller by a shift $\Delta$ than the corresponding simulated AR(1) noise eigenvalues due to the fact that real data contain both signal and noise (Cordes and Nandy, 2006). The shift $\Delta$ can be determined from the tail spectrum, and the eigenvalue spectrum of the simulated AR(1) noise data can be properly adjusted. Thus, the adjusted eigenvalue spectrum of the simulated AR(1) noise data is compared to the eigenvalue spectrum of corresponding fMRI data.

Step 4. Estimate the intrinsic dimensionality. After adjusting the eigenvalue spectrum of the simulated AR(1) noise data, the number of eigenvalues of real fMRI data that are larger than the simulated noise eigenvalues defines the dimension of the signal space. We can get the estimation of the intrinsic dimensionality by counting the number of eigenvalues of real fMRI data that are larger than the simulated noise eigenvalues from $k=1$ to the first intersection between the adjusted eigenvalue spectrum of simulated noise data and the eigenvalue spectrum of real fMRI data.

\section{Spatiotemporal Lyapunov Exponent}

A method (Ricard and Jordi, 1995) based on coupled map lattices (CML), which widely used as models of spatiotemporal chaos in physical, chemical and biological systems, is introduced to evaluate numerically a Spatiotemporal Lyapunov Exponent when very short time series are obtained from a spatially distributed dynamical system. This method has been proved equal to detecting nonlinear attribution of a spatial evolution with common short time series.

A dynamical system is given by a set of nonlinear equations as follow:

$x_{n+1}^{j}(\mathbf{k})=F_{\mu}^{j}\left(\mathbf{x}_{n}(\mathbf{k})\right)+C_{\gamma}^{j}\left(\mathbf{x}_{n}(\mathbf{k})\right)$

where $j=1, \ldots, s, \mathbf{x}=\left(x_{n}^{1}, \ldots, x_{n}^{s}\right)$ and $F_{\mu}^{j}(\mathbf{x}), C_{\gamma}^{j}(\mathbf{x}) \in C^{2}(U) . U$ is a compact set and $U \subset R^{s}$. This set of maps is then defined on a twodimensional lattice

$\Lambda^{2}(L)=\{\mathbf{k}=(\xi, \beta) \mid 1 \leq \xi, \beta \leq L\}$

If we have a time series defined as the set:

$\Gamma^{j}(\mathbf{k})=\left\{X_{1}^{j}(\mathbf{k}), \ldots, x_{M}^{j}(\mathbf{k})\right\} \quad \forall \mathbf{k} \in \Lambda^{2}(L)$

using the lagging method, we can construct new $m$-dimensional sets as follow:

$\Gamma_{m}^{j}(\mathbf{k})=\left\{x_{1}^{j}(\mathbf{k})=\left(x_{i}^{j}(\mathbf{k}), \ldots, x_{i+m-1}^{j}(\mathbf{k})\right)\right\}$

where $i=1, \ldots, M-m+1$ and $m$ is an embedding dimension. Now we consider the global set defined as the union of all local orbits:

$\Gamma_{m}(\Lambda)=\bigcup_{\mathbf{k} \in \Lambda(L)} \Gamma_{m}^{j}(\mathbf{k})$

This set is then constructed by $(M-(m-1)) L^{2}$-points.

For each vector $X_{i}^{j}(\mathbf{k})$, we search those lattice points $\mathbf{h} \in \Lambda^{2}(L)$, $(\mathbf{h} \neq \mathbf{k})$ such that the inequality

$\left\|X_{i}^{j}(\mathbf{k})-X_{i}^{j}(\mathbf{h})\right\|=\left[\sum_{u=i}^{i+m-1}\left(x_{u}^{j}(\mathbf{k})-x_{u}^{j}(\mathbf{h})\right)^{2}\right]^{1 / 2}<\varepsilon$ 
can hold. Here $\varepsilon$ is the maximum initial separation. Then the Spatiotemporal Lyapunov Exponent will be evaluated by:

$\lambda_{s}(m)=\frac{1}{N_{p}} \sum_{i=1}^{M-m} \sum_{(\mathbf{k}, \mathbf{h})} \ln \left[\frac{\left\|X_{i+1}^{j}(\mathbf{k})-X_{i+1}^{j}(\mathbf{h})\right\|}{\left\|X_{i}^{j}(\mathbf{k})-X_{i}^{j}(\mathbf{h})\right\|}\right]$

where $N_{\mathrm{p}}$ is the total number of $<\mathbf{k}, \mathbf{h}>$ pairs.

Here, STLE can be regard as an index of the spatiotemporal coupling between voxels of fMRI data. The Lyapunov exponents are related to the average rates of divergence of nearby trajectories in phase space. System that exhibits a limit cycle or torus as its attractor exhibits no positive Lyapunov exponents. When at least one Lyapunov exponent is positive, then the system at hand is chaotic, and the initial sphere will evolve to some complex ellipsoid structure reflecting the exponential divergence of nearby initial conditions along at least one direction on the attractor.

In some sense, correlation dimension is often used to indicate what a system is not, too. Noise has an infinite correlation dimension and thus has a correlation dimension equal to the embedding dimension. On the other hand, a limit cycle or torus system has integer correlation dimension. Therefore, a finite and non-integer correlation dimension is an indicator that the underlying system is dominated neither by noise nor by a limit cycle or torus orbit.

\section{Surrogate data}

The most popular method used to identify the nonlinearity of raw data is a statistical approach utilizing the technique of surrogate data (Schreiber and Schmitz, 2000; Hegger and Kantz, 1999; Kantz and Schreiber, 1997). Since this technique is introduced methodologically into nonlinear dynamics, it has been widely used through nonlinear data processing (Stam, 2005; Lee et al., 2001; Stam et al., 1995). Surrogate data are artificially generated that mimic some properties of the data under study. They can be gained by many methods.

In order to test the null hypothesis that the data are independent random numbers, surrogate times series can be simply obtained by randomly shuffling the measured data (Kantz and Schreiber, 1997; Schreiber and Schmitz, 2000; LaConte et al., 2004), which take on exactly the same values as the data, just in random temporal order. If there is significant difference of the nonlinear property between the data and their shuffles, the hypothesis of independent random numbers can be rejected.

Another surrogate for nonlinearity test is designed to test the null hypothesis that the signal consists of linearly filter Gaussian noise (Lee et al., 2001). The surrogate data should have the same power spectrum and autocorrelation function as the raw data under study (Schreiber and Schmitz, 2000). It can be gained by randomizing the phase of original raw signal (Schreiber and Schmitz, 2000; Lee et al., 2001). Firstly, compute the Fourier spectrum of the raw data using Fourier transformation (FT). The Fourier spectrum is complex amplitude at each frequency. Secondly, randomize the phase of the complex amplitude, i.e. each complex amplitude is multiplied by $\mathrm{e}^{\mathrm{i} \varphi}$, where $\varphi$ is independently chosen from $[0,2 \pi]$ for each frequency. Then a new complex spectrum in the frequency domain, which has the same absolute values as the Fourier spectrum of the raw data, can be obtained. Thirdly, generate the surrogate data. The new Fourier spectrum is transformed back into the time domain by inverse Fourier transform (IFT) and the result is the surrogate data, which is a realization of linear stochastic process with the same power spectrum as the raw data. If there is significant difference of the nonlinear property between the data and their surrogate data, the hypothesis of linearly filter Gaussian noise can also be rejected.

For surrogate test, multivariate data can also be treated very much the same way as scalar time series (Schreiber and Schmitz, 2000). Moreover, we can decide either to keep or to destroy their crosscorrelations between two time series. If all time series are applied the same permutations, their cross-correlations can be retained.

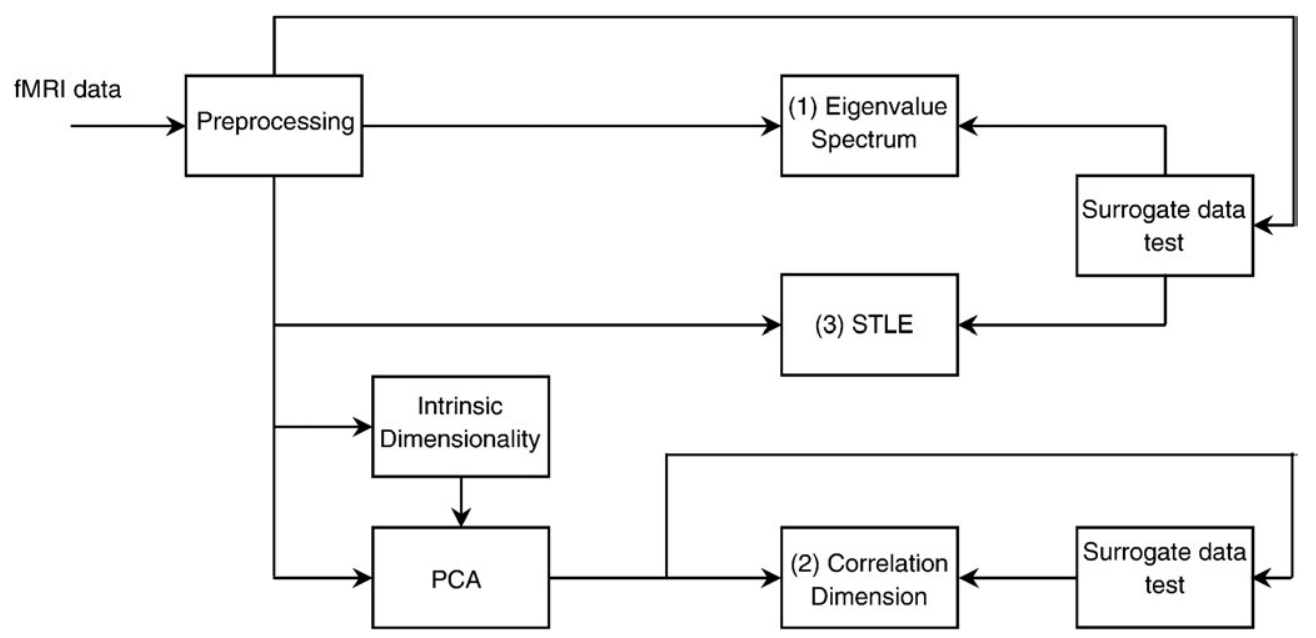

Fig. 3. List of procedures applied to the fMRI data. After standard slice-time correcting and realigning, there were three process parts for fMRI data analysis. (1) Eigenvalue spectra analysis. In this part, the eigenvalue spectra for the covariance matrix of the fMRI datasets were analyzed (2) Correlation dimension analysis. The intrinsic dimensionalities of the fMRI data were estimated by the method based on AR(1) noise model which the AR(1) coefficient was estimated by cubic spline interpolation. After extracting important modes of activity from fMRI data by use of Principal Component Analysis according to the estimated intrinsic dimensions, phase-space was reconstructed by the time-delay embedding for the principal components and then the correlation dimension based on multivariate series was calculated. (3) Spatiotemporal Lyapunov Exponent analysis. In this part, Spatiotemporal Lyapunov Exponents, bases on coupled map lattices (CML), were also calculated to fMRI data. In order to test the nonlinearity in the fMRI, surrogate data generated from fMRI data were introduced in any analysis part. 

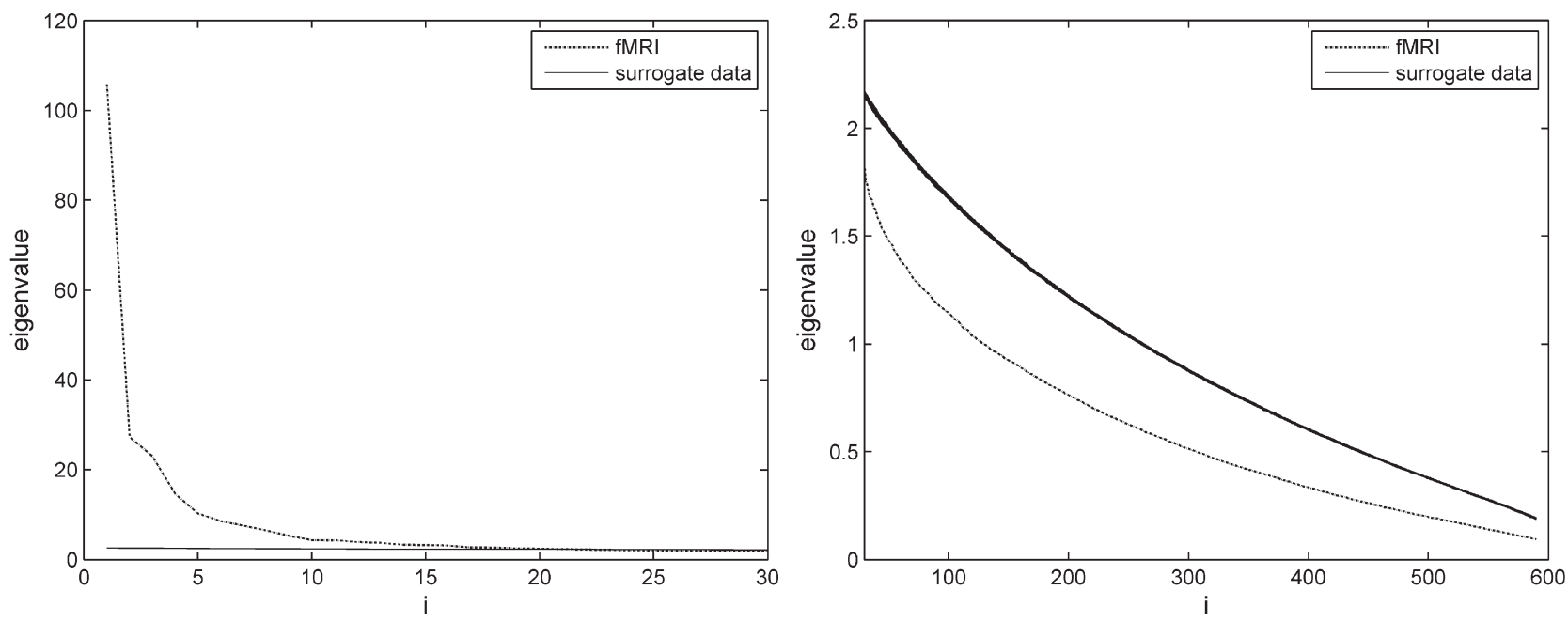

Fig. 4. Eigenvalue spectrum for real resting-state fMRI data of a single subject using all 600 time frames (dashed line). The solid lines correspond to the eigenvalue spectra of its 116 surrogate data generated by randomizing its temporal order. The left shows the very first 30 eigenvalues. The right shows the rest. The last 10 eigenvalues are not shown.

\section{Data acquisition and analysis}

Single-shot gradient echo planar imaging (EPI) data were acquired from nine healthy volunteers in resting state with closing eyes, stopping thinking if any idea came up. The data were obtained on a 1.5T PHILIPS MEDICAL SYSTEMS Gyroscan NT scanner $(\mathrm{TR}=700 \mathrm{~ms}$, Flip Angle $=70 \mathrm{deg}$ and $\mathrm{FOV}=23 \mathrm{~cm}$, with 5 transection slices covering the visual cortex and other 5 transection slices covering the motor cortex, $5 \mathrm{~mm}$ slice thickness, matrix size: $64 \times 64$ ). After discarding initial scans (to allow for magnetic saturation effects) each time series was comprised of 600 scan images.

The data were preprocessed using SPM2 (http://www.fil.ion. ucl.ac.uk/spm/software/spm2) software. All time-series were slicetime corrected, which corrected differences in image acquisition time between slices, and realigned, which corrected for movementrelated effects. A mask containing only brain voxels was generated by a threshold. In order to retain as much information as possible, no further preprocess was done.

Fig. 3 summarizes the analysis steps to detect the nonlinearity of the resting-state fMRI data.

\section{Results and Discussion}

Eigenvalues of covariance matrix for real fMRI data (Figs. 4, 5)

The eigenvalue spectra of the covariance matrix $R$ for real fMRI data were firstly calculated. To obtain estimates of significance, two kinds of surrogate data were generated as described in above paragraph. Fig. 4 shows the eigenvalue spectrum for real restingstate fMRI data of a single subject and the eigenvalue spectra of its 116 surrogate data $(p<0.01)$ generated by randomizing their temporal order. The left shows very first 30 eigenvalues of the eigenvalue spectra. The right shows the rest of eigenvalues. The last
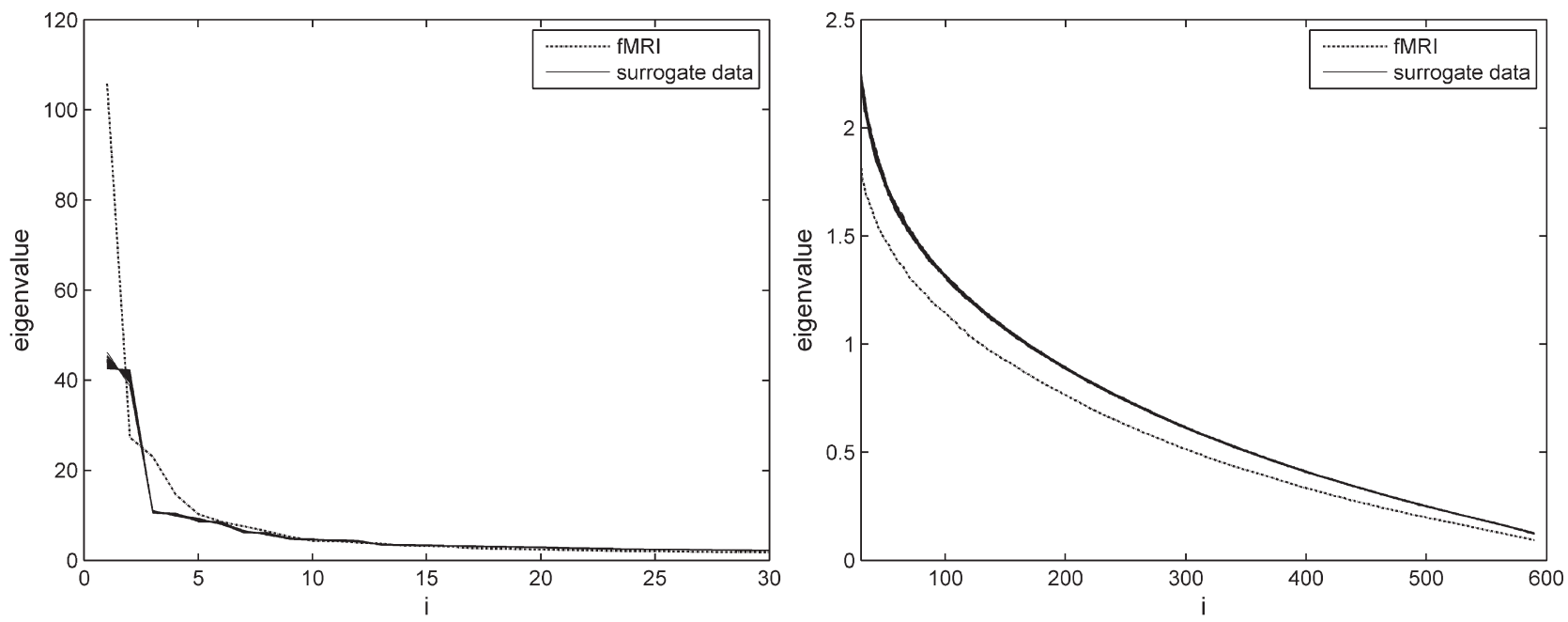

Fig. 5. Eigenvalue spectrum for real resting-state fMRI data of a single subject using all 600 time frames (dashed line). The solid lines correspond to eigenvalue spectra of its 116 surrogate data generated by Fourier transform with the same power spectrum as the raw data. The left shows the very first 30 eigenvalues. The right shows the rest. The last 10 eigenvalues are not shown. 

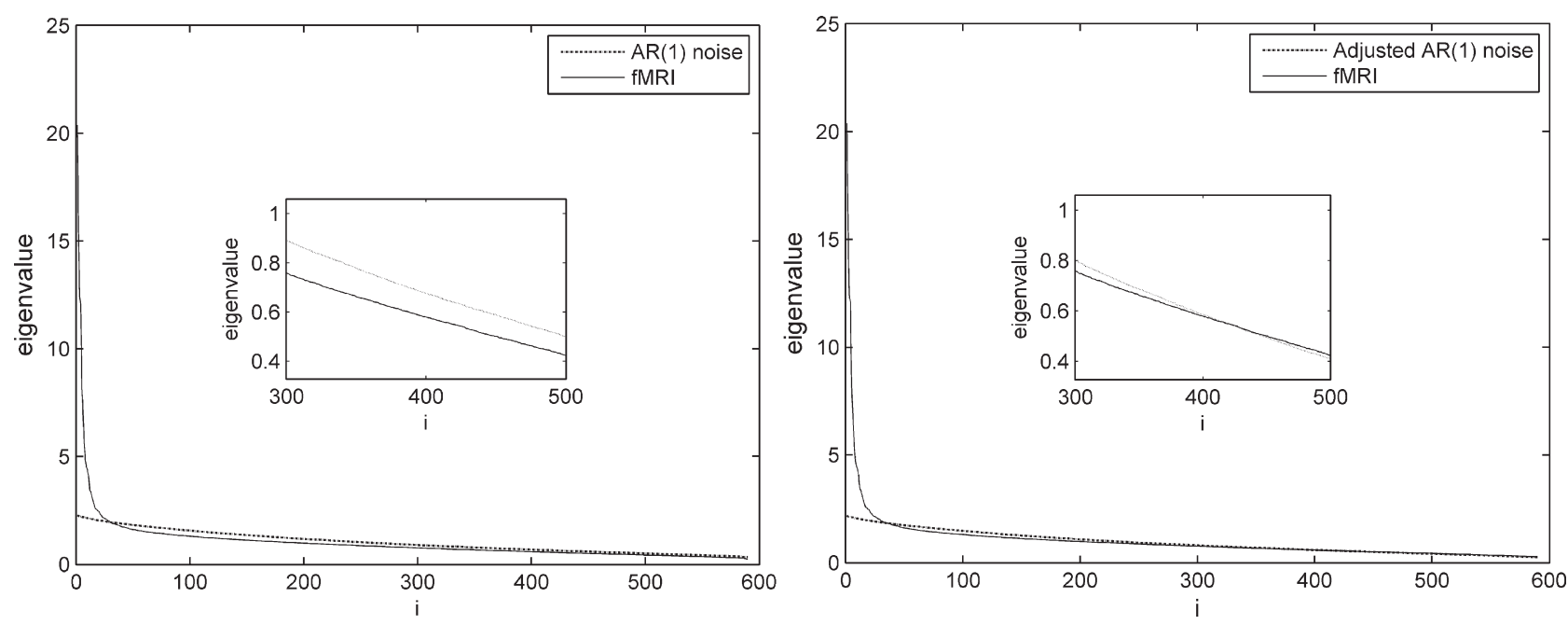

Fig. 6. Eigenvalue spectra for real resting-state fMRI data of a single subject using all 600 time frames (solid line). The dashed line of left plot corresponds to eigenvalue spectrum of pure AR(1) noise for $M=600$ where the AR(1) parameter was estimated from data using the method introduced in the paper. The dashed line of right plot corresponds to its tail-adjusted eigenvalue spectrum of pure AR(1) noise for $M=600$. The last 10 eigenvalues are not shown. The central plot of the figure is the zooming of detail in a smaller area of $i \in[300,500]$.

10 eigenvalues are not shown in the figure. Fig. 5 shows the eigenvalue spectrum for real resting-state fMRI data of the same subject and the eigenvalue spectra of its 116 surrogate data generated by randomizing the phase of the Fourier spectrum. The left shows the very first 30 eigenvalues of the eigenvalue spectra. The right shows the rest. The last 10 eigenvalues are also not shown in the figure.

The distribution of the eigenvalues is directly related to the amount of correlations of the multivariate data set (Müller and Baier, 2005). If all $N$ time series are noncorrelated, the nondiagonal elements of the covariance matrix $R$ tend to zero when the time of the data tends to infinity. In that case the spectrum of $R$ is completely degenerate and all the diagonal elements $\lambda_{\mathrm{i}}=1 \forall i$. If all $N$ time series are perfectly correlated, all the elements of $R$ tend to one. Only one eigenvalue of this matrix is nonzero, i.e. $\lambda_{\max }=N$. For real fMRI data, the distribution of the eigenvalues is between these two extremes. It suggests that real fMRI data is neither perfectly noncorrelation, nor perfectly correlation. In Fig. 4, the surrogate data generated by randomizing temporal order, which all the eigenvalues are distributed around 1, are almost noncorrelation between $N$ time series and close to random noise. Because of significant differences of eigenvalue spectra between real fMRI and their surrogate data, fluctuations of resting-state fMRI data of the human brain, therefore, cannot be fully attributed to random noise. It is also shown that there is some correlation between all $N$ time series of fMRI data. It is the correlation that is usually used to detect functional connection in resting-state human brains (Luca et al., 2006; Lahaye et al., 2003; Hampson et al., 2002; Lowe et al., 1998).

\section{Intrinsic dimensionality estimation (Table 1, Fig. 2, Fig. 6)}

After constructing the simulated pure AR(1) noise data with the same time dimension and voxel number as fMRI data, the typical coefficients $a(\phi)$ and $b(\phi), \phi \in[0,0.5]$, were constructed. Because the tail eigenvalue spectrum of the sample covariance matrix of fMRI data was also fitted to $\lambda(k)=a_{\gamma} e^{-b_{\gamma} k}$, the coefficients $a_{\gamma}$ and $b_{\gamma}$ corresponding to the fMRI data were obtained. Assuming that the tail eigenvalues of the covariance matrix of fMRI data would not have any contribution for the signals, the proper $\phi_{\gamma}$ at the obtained coefficient $b_{\gamma}$ corresponding to fMRI data, which is also the $\operatorname{AR}(1)$ coefficient, can be estimated from the plot of $b(\phi)$ against $\phi$ using cubic spline interpolation. Fig. 6 (left plot) shows the eigenvalue spectrum of fMRI data for one subject together with the eigenvalue spectrum of simulated AR(1) noise generated by using the estimated $\phi_{\gamma}$ corresponding to the same fMRI data. From Fig. 6 (left plot), it is shown that the tail eigenvalues for real fMRI data will always be smaller by a shift $\Delta$ than the corresponding simulated AR(1) noise eigenvalues. The eigenvalue spectrum of real fMRI data for the same subject together with the adjusted eigenvalue spectrum of AR (1) noise is also shown in Fig. 6 (right plot). The intrinsic dimension was estimated by counting the number of eigenvalues of real fMRI data that are larger than the corresponding adjusted noise eigenvalues from $k=1$ to the first intersection between the adjusted noise eigenvalue spectrum and the real fMRI data eigenvalue spectrum.

The intrinsic dimensionalities of real fMRI data of nine subjects could be estimated by the above method. The results are shown in Table 1.

Table 1

The intrinsic dimensionality of fMRI data

\begin{tabular}{llllllllll}
\hline & Subject 1 & Subject 2 & Subject 3 & Subject 4 & Subject 5 & Subject 6 & Subject 7 & Subject 8 & Subject 9 \\
\hline Motor slices & 18 & 22 & 23 & 27 & 20 & 21 & 21 & 32 & 24 \\
Visual slices & 27 & 24 & 37 & 35 & 39 & 37 & 38 & 35 & 33 \\
\hline
\end{tabular}

Note. Motor slices describe all transection slices covering the motor cortex and visual slices all transection slices covering the visual cortex. 
In order to compromise between essential information preservation and appropriate calculation quantity, there is an important problem how many components should be taken from fMRI data for estimating the correlation dimension. The intrinsic dimensionalities of the real fMRI data in resting-state of the human brain, which are shown in Table 1, indicate that all numbers of essential components are less than 40 for nine subjects. For example, the intrinsic dimensionality is 18 for the first subject. Namely, 18 components are enough to extract important modes of activity from fMRI data for the first subject. From a point of view which information of fMRI data is retained, it is enough to estimate the correlation dimension using these 18 components too. However, if there is no exceptionally large data set available, the correlation dimension estimate cannot be achieved because of "boundary effects (edge effects)" (Raab and Kurths, 2001, Galka, 2000) and "finite-size effects" (Raab et al., 2006). The boundary effects result in decreasing of $d_{2}(\varepsilon, m)$ for large distances $\varepsilon$, and the finite-size effects make awkward or tremendous fluctuations of $d_{2}(\varepsilon, m)$ for small distances $\varepsilon$. These two kinds of effects will make the scaling region narrowed, even disappeared, so that no estimation of the correlation dimension can be obtained. On the other hand, for estimating the correlation dimension, the larger the amount of data is, the better the result is, but the larger the calculation quantity is as well. Too much amount of data will result in horrible calculation quantity. Because the maximum intrinsic dimension of nine subjects is 39 and less than 40 , therefore, it is reasonable to calculate the correlation dimension using $40 \mathrm{com}$ ponents extracted from fMRI data of each subject. It assures not only including all the necessary components of fMRI data and having enough large data set for estimating the correlation dimension, but also having proper calculation quantity.

\section{Correlation dimension estimation (Table 2, Fig. 7-9)}

Before estimating the correlation dimension, PCA was implemented to extract a few components from fMRI data. Based on the confirmed intrinsic dimensionalities, 40 properly components were extracted from real fMRI data (the first component was excluded because it did not represent those involved in the functional dynamics but the variance due to drift of the baseline). The extraction of useful principal components not only retains the
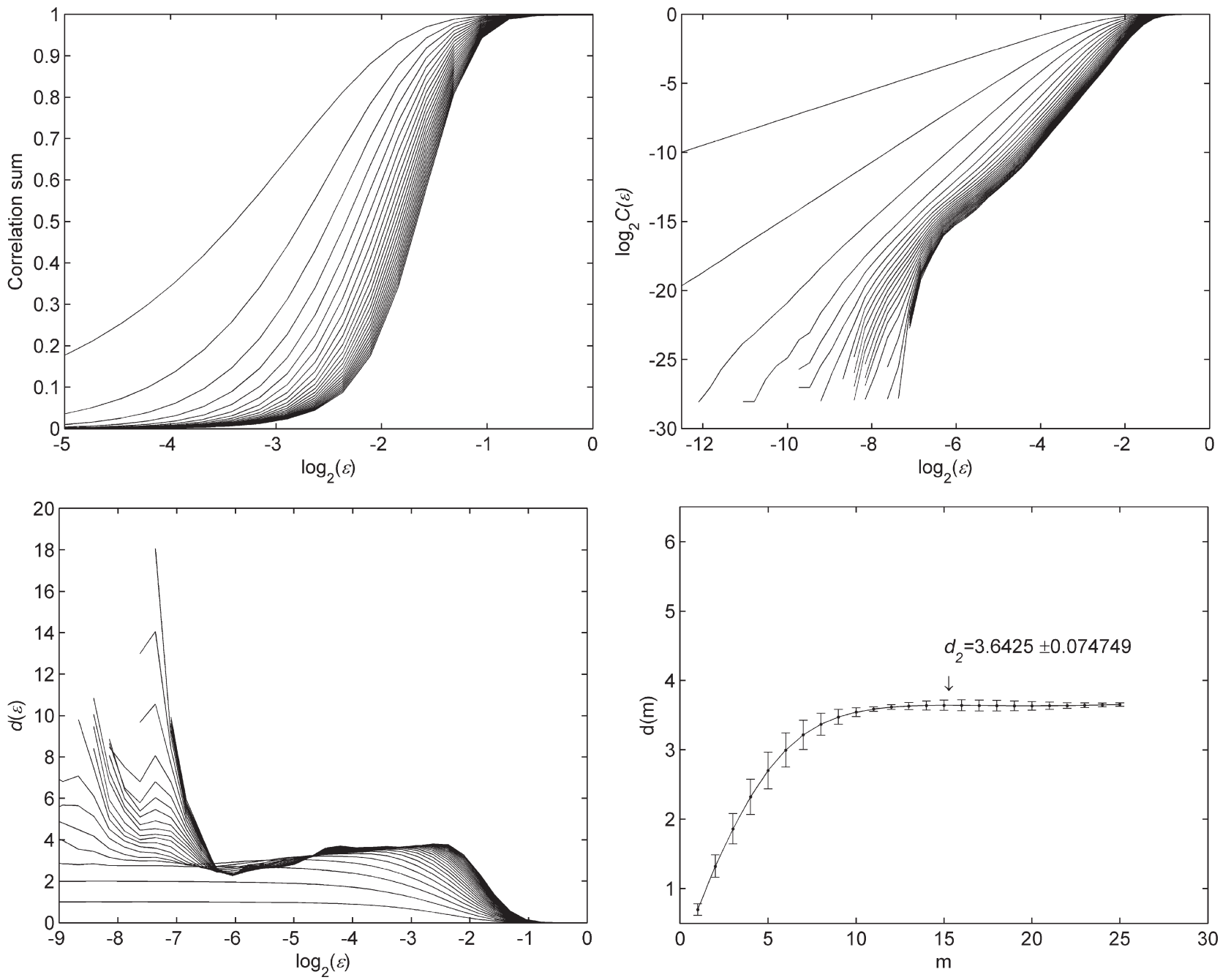

Fig. 7. The upper left is the correlation sums for the principal components of resting-state fMRI as the embedding dimension $m$ is from 2 to 25 . The upper right is the plot of $\log _{2} C(\varepsilon)$ against $\log _{2} \varepsilon$ at the same scope of embedding dimensions. The lower left is the local slopes $d[\varepsilon]$ against $\log _{2} \varepsilon$. The $\operatorname{lower}_{\text {right is }}$ the correlation dimensions with the embedding dimensions. 
Table 2

The correlation dimension of fMRI data

\begin{tabular}{|c|c|c|c|c|c|c|c|c|c|}
\hline & Subject 1 & Subject 2 & Subject 3 & Subject 4 & Subject 5 & Subject 6 & Subject 7 & Subject 8 & Subject 9 \\
\hline Motor slices & $4.19 \pm 0.11$ & $3.63 \pm 0.03$ & $5.60 \pm 0.19$ & $6.46 \pm 0.37$ & $5.73 \pm 0.24$ & $9.34 \pm 0.24$ & $8.65 \pm 0.27$ & $8.81 \pm 0.07$ & $8.57 \pm 0.33$ \\
\hline Visual slices & $4.44 \pm 0.07$ & $3.70 \pm 0.08$ & $4.78 \pm 0.11$ & $4.86 \pm 0.02$ & $4.46 \pm 0.04$ & $3.71 \pm 0.02$ & $2.70 \pm 0.11$ & $3.64 \pm 0.07$ & $4.51 \pm 0.06$ \\
\hline
\end{tabular}

Note. Motor slices describes all transection slices covering the motor cortex and Visual slices describes all transection slices covering the visual cortex.

principal information including in fMRI data, but also reduces both computational load and noise level in the signals. Out of the extracted principal component set, a phase space was reconstructed with a time-delay embedding scheme for embedding dimensions $m$ from 2 to 25 , as well as proper delay times $\tau$. Each subject has a delay time $\tau$ itself, which was determined by using mutual information method (Kantz and Schreiber, 1997), and all components have the same $\tau$ for one subject. With the delay vectors constructed from 24000 data points (40 components $\times 600$ time points), the correlation dimension could be estimated.

Fig. 7 is the plots of correlation sum $C(\varepsilon)$ against $\log _{2}(\varepsilon), \log _{2} C$ ( $\varepsilon$ ) against $\log _{2}(\varepsilon), d(\varepsilon, m)$ against $\log _{2}(\varepsilon)$, and $d(m)$ against $m$ for fMRI data of one subject. At reconstructed phase space with embedding dimensions $m=2$ to 25 , the plots indicate power law behavior of the correlation sum with most of the length scale $\varepsilon$. A scaling behavior is apparent as shown in the plot of local slopes $[d(\varepsilon$, $m)]$ against $\log _{2}(\varepsilon)$. The values for $d(m)$ can be read off in scale region. From the plot of local slopes $[d(\varepsilon, m)]$ against $\log _{2}(\varepsilon)$, it can also be shown that statistical fluctuations (finite-size effects and noise) distort the scaling behavior below this length scale, and the decreases (boundary effects) destroy the scaling behavior above this length scale. At sufficiently high embedding dimensions, a saturation value appears from this scaling region as shown in Fig. 7 (lower right). The saturation value is defined as the correlation dimension $d_{2}$.

Table 2 shows the correlation dimensions of resting state fMRI data of nine subjects. All subjects have the nature of non-integer correlation dimensions.

To test nonlinearity, two kinds of multivariate surrogate data were generated from the principal component sets, as described in above paragraph, and their correlation dimensions were calculated as well. Fig. 8 shows the local slopes $d(\varepsilon, m)$ against $\log _{2}(\varepsilon)$ for one of 20 surrogate data $(p<0.05)$. The comparison of correlation dimensions between fMRI data and two kinds of surrogate data is shown in Fig. 9. For fMRI data, there are significant scale region and saturation of $d(m)$ as the embedding dimension $m$ increases. But for two kinds of surrogate data, there are no scale region and saturation of $d(m)$.

The apparent differences of correlation dimensions between fMRI data and their surrogate data, as well as fractal correlation dimensions shown in Table 2, indicate that the resting state fMRI signals cannot fully attribute to: (1) white noise; (2) filtered noise; (3) linear stochastic process. Therefore it can be concluded out that there is nonlinear fractal behavior in fMRI signals and dominant deterministic dynamical structure governing the human brain.

Although estimation of the correlation dimensions can give us useful measures to expect the order parameters of brain dynamics (Dhamala et al., 2002), successful correlation dimension estimation requires stationary time series, sufficient amount of data set with noise-free, appropriate choice of the parameters for phase space reconstruction. Unfortunately, for fMRI data, these conditions can not be fulfilled completely, and the estimates of dimension may not reflect the true dimensions of the brain dynamics. However, it is demonstrated (Galka, 2000) that the "traditional" correlation dimension estimation may already yield reasonable information about the temporal evolution of the dynamics, and this fact implies that the correlation dimension in various conditions encountered in medical and physiological research at least contain qualitatively useful information about the underlying process. Here, it can be considered that the estimated correlation dimensions contain rea-
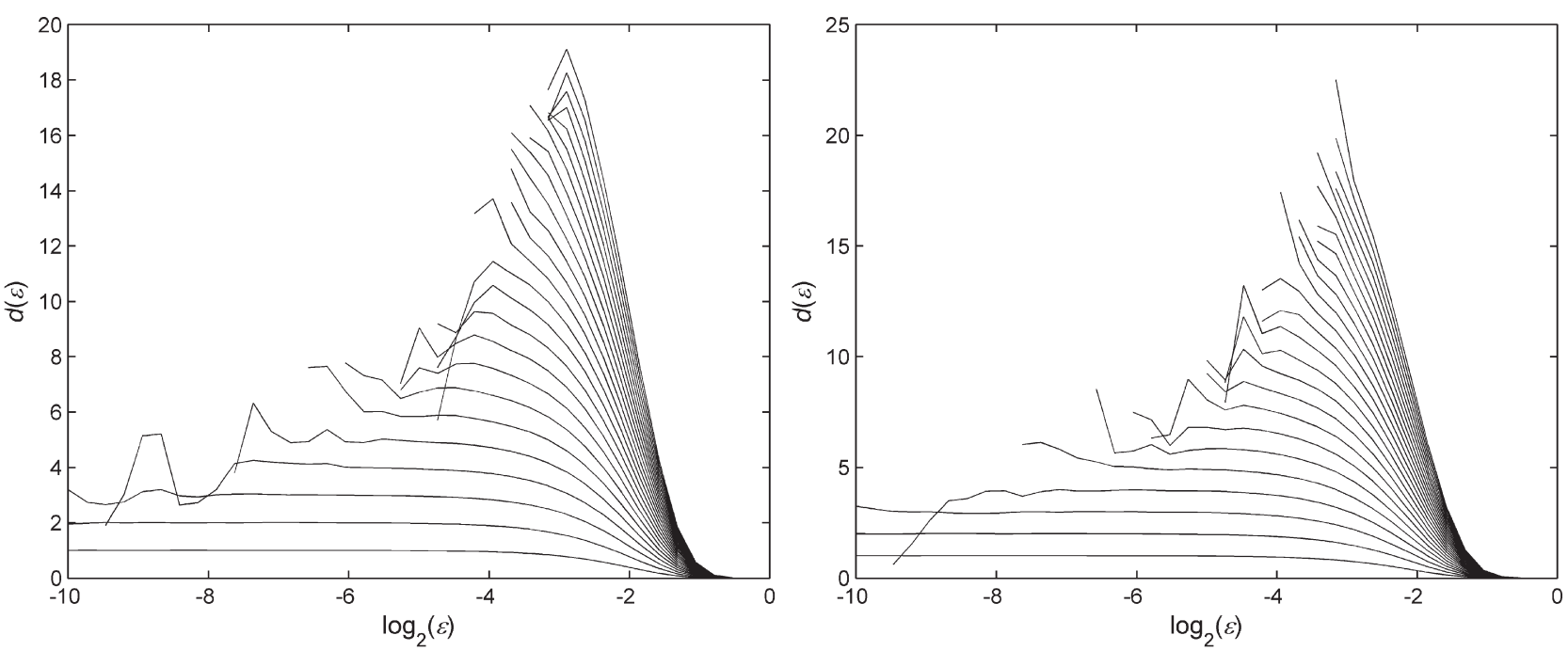

Fig. 8. The left is the local slopes $d[\varepsilon]$ against $\log _{2} \varepsilon$ of surrogate data gained by permuting as the embedding dimension $m$ is from 2 to 25 . The right is the local slopes $d[\varepsilon]$ against $\log _{2} \varepsilon$ of surrogate data gained by randomizing their phases in the Fourier domain. There is apparently no scale region for the multivariate surrogate data. 


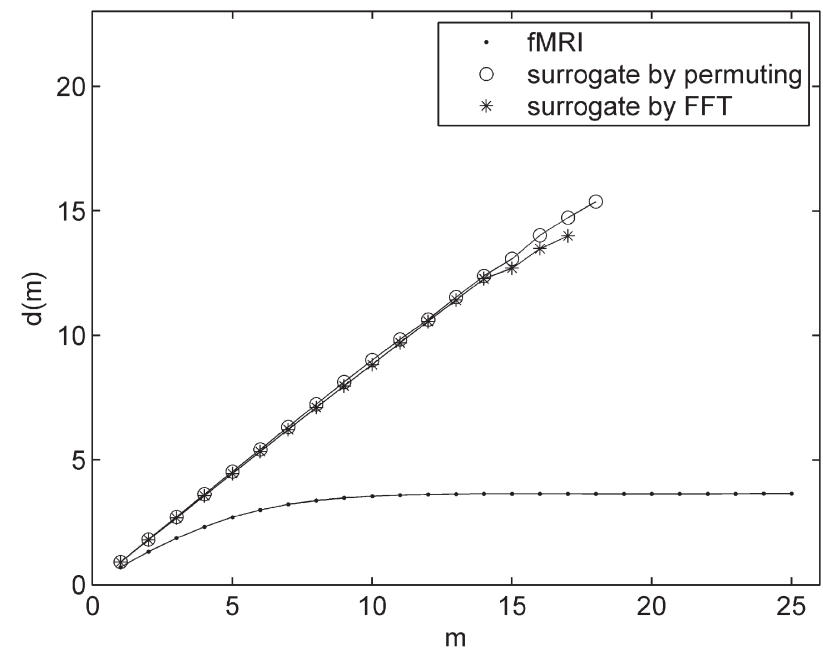

Fig. 9. The comparison of the correlation dimension of $\mathrm{fMRI}$, surrogate data generated by permuting and surrogate data generated by Fourier transform. For fMRI data, there is a significant saturation of $d(m)$ as embedding dimension $m$ increases. But for two kinds of surrogate data, there is no saturation of $d(m)$.

sonable and useful information about brain dynamics of resting state and spatiotemporal nonlinearity in rest-state fMRI data of the human brain is detected.

Spatiotemporal Lyapunov Exponent analysis (Table 3, Fig. 10)

The STLEs were calculated to the slices numbered by 2, 3, and 4 of the resting state fMRI data for the human brain. The embedding dimension $m=8$, and $\varepsilon=0.02$. The STLEs of nine subjects are shown in Table 3. The most important characteristic is that all the STLEs are positive.

To test the nonlinearity of coupling between voxels of fMRI data, the time evolutions of the STLEs were calculated to 25 surrogate data $(\mathrm{p}<0.05)$ generated by two methods. One kind of surrogate data was obtained by randomly shuffling the measured data, and keeping simultaneously their cross-correlations between voxels in order to retain the information of linear coupling. The other kind of surrogate data was gained by randomizing the phase of the Fourier spectrum of real fMRI data, and keeping simultaneously the cross-correlations between voxels in order to maintain their linear correlations, too. In fact, the second sets of surrogate data retain the same covariance matrix as the corresponding fMRI data. They can be used to test not only the nonlinear correlations between voxels, but also the nonlinearity in each voxel time series itself. The results for one subject were shown in Fig. 10. The significant differences of the STLEs between fMRI data and their surrogate data, as well as the positive STLEs (shown in Table 3) which characterize the nonlinear chaotic property of fMRI data, indicate that resting state fMRI signals cannot fully attribute to: (1) white noise; (2) filtered noise; (3) linear stochastic process; (4) linearly coupled.

As we all know, the contraction of the heart is approximately periodic, i.e. the cardiac rhythm appears to be quasi-periodic, and many biological rhythms (including cardiac rate and respiration) are best represented mathematically as limit cycle oscillations in differential equations (Glass and Mackey, 1988). Therefore, breathing and cardiac activity in resting-state usually appear some properties of the limit cycle or torus attractors. As mentioned above, noise has an infinite correlation dimension and a limit cycle or torus system has an integer correlation dimension. On the other hand, a system that exhibits a limit cycle or torus as its attractor has no positive Lyapunov Exponents. The results of finite correlation dimensions significantly lower than the embedding dimensions, non-integer correlation dimensions (Table 2) and positive STLEs (Table 3) suggest that the underlying system is dominated by neither noise nor a limit cycle or torus orbit. In other words, the nonlinearity of fMRI data cannot be raised by respiratory movement and cardiac activity. It is the intrinsic property in the restingstate human brain.

Through analysis above, it is considered that resting-state fMRI signals of the human brain have spatiotemporal nonlinear properties. Fluctuations at very low frequencies $(0.1 \mathrm{~Hz})$ presented in resting state fMRI signals may be an inherent model of basal neural activation in the human brain, and cannot be full attributed to noise. This activity exhibits striking patterns of coherence (Raichle, 2006; Thirion et al., 2006) within known networks of specific neurons in the human brain in the absence of observable behaviors.

\section{Conclusion}

In this work, the spatiotemporal nonlinearity in resting-state fMRI data of the human brain was detected by nonlinear dynamics methods. Eigenvalue spectra, correlation dimensions and Spatiotemporal Lyapunov Exponents were calculated to demonstrate the spatiotemporal nonlinearity in resting-state fMRI data of the human brain.

Eigenvalue spectra analysis results of the covariance matrix for resting-state fMRI data in the human brain suggest that fMRI data are neither perfectly noncorrelation, nor perfectly correlation. Because of significant differences of eigenvalue spectra between real fMRI data and their surrogate data, fluctuations of resting-state signals in the human brain, therefore, cannot be fully attributed to noise, and there is a determined correlation between all time series of fMRI data.

Table 3

The STLE of fMRI data in resting state brain

\begin{tabular}{|c|c|c|c|c|c|c|c|c|c|}
\hline & Subject 1 & Subject 2 & Subject 3 & Subject 4 & Subject 5 & Subject 6 & Subject 7 & Subject 8 & Subject 9 \\
\hline Slice2 of SCMC & 0.1782 & 0.1503 & 0.2283 & 0.2774 & 0.2030 & 0.1708 & 0.2133 & 0.1621 & 0.2451 \\
\hline Slice 3 of SCMC & 0.2297 & 0.1505 & 0.2150 & 0.2694 & 0.2361 & 0.1834 & 0.2261 & 0.1940 & 0.2540 \\
\hline Slice4 of SCMC & 0.2259 & 0.1516 & 0.2177 & 0.2562 & 0.2520 & 0.2601 & 0.2488 & 0.1889 & 0.2306 \\
\hline Slice2 of SCVC & 0.1754 & 0.1050 & 0.2206 & 0.2494 & 0.2784 & 0.1694 & 0.2523 & 0.1971 & 0.2571 \\
\hline Slice3 of SCVC & 0.2006 & 0.1456 & 0.2263 & 0.2499 & 0.2571 & 0.1820 & 0.2514 & 0.1930 & 0.2614 \\
\hline Slice4 of SCVC & 0.1750 & 0.1749 & 0.2246 & 0.2428 & 0.2764 & 0.1978 & 0.2347 & 0.2107 & 0.2593 \\
\hline
\end{tabular}

Note. SCMC means slice covering the motor cortex and SCVC means slice covering the visual cortex. 

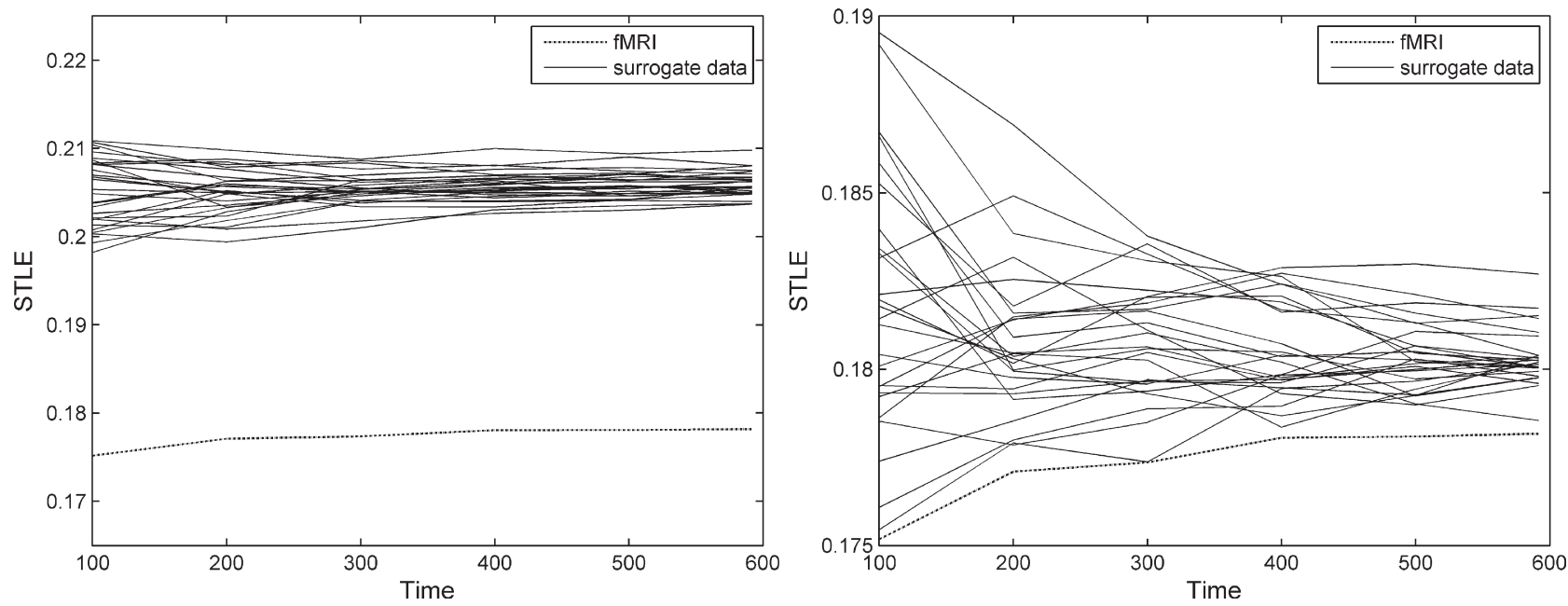

Fig. 10. The STLEs evolved with time. The left shows STLEs of fMRI data and its 25 surrogate data generated by permuting. The right shows STLEs of fMRI data and its 25 surrogate data generated by Fourier transform. There is apparent difference between fMRI and its surrogate data.

By simulating, adjusting, and comparing eigenvalue spectra of proper AR(1) noise with the corresponding real fMRI data, the intrinsic dimensionality is estimated. Then the intrinsic dimensionality is used to extract the first few principal components from real fMRI data using PCA, which will preserve the correct phase dynamics, while reducing both computational load and noise level of the data.

In the phase-space, which was reconstructed using the timedelay embedding method for their principal components, the correlation dimension of spatiotemporal series was estimated by use of the Grassberger-Procaccia algorithm. Scaling behaviors about $d(\varepsilon, m)$ are apparent and the saturations of $d(m)$ can be read off, which have the nature of non-integer correlation dimensions for all subjects. On the other hand, the STLEs were calculated by using coupled map lattice methods to resting state fMRI of the human brain, and are positive for all subjects.

To test the nonlinearity, two kinds of multivariate surrogate data were generated using randomly shuffling in time domain and phase-scrambling in Fourier space for resting state fMRI data and correlation dimensions and STLEs were also calculated for two kinds of surrogate data. By comparison of correlation demensions and STLEs, the significant differences between fMRI data and two kinds of surrogate data lead us to conclude out that resting state fMRI signals cannot fully attribute to: (1) white noise; (2) filtered noise; (3) linear stochastic process; (4) linearly coupled. Therefore, we could convince that the spatiotemporal nonlinear dynamics property in fMRI signals is detected, and there exists the deterministic nonlinear behavior in the resting state human brain. Fluctuations at very low frequencies $(0.1 \mathrm{~Hz})$ presented in resting state fMRI signals may be an inherent model of basal neural activation of the human brain.

Further work will study the nonlinearity and the interrelation of special regions of the human brain to reflect pathologic and/or physiologic alterations. In order to test nonlinearity in more detail, the surrogate data generated by wavelet analysis method (Sendur et al., 2007; Friman and Westin, 2005; Bullmore et al., 2004; Breakspear et al., 2003) will be used. We believe that measures of nonlinearity with the spatial distribution of activity will ultimately yield intensive understanding into the function of the human brain.

\section{Acknowledgments}

The authors acknowledge the support of the National Natural Science Foundation of China (Grant No. 50577057) and Key Project of Humanities and Social Sciences, Ministry of Education of China (No. 07JZD0029).

\section{Appendix}

\section{The shift $\Delta$}

Suppose that the real data comprise $p$ signal components and their tail eigenvalues of the covariance matrix can be fitted by an exponent function $\lambda_{0}(k)=a \mathrm{e}^{-b k}$, and $k=p+1, p+2, \ldots, M$; the $\operatorname{AR}(1)$ coefficient $\phi_{\gamma}$ can then be estimated using the method based on AR(1) noise model. The corresponding simulated AR(1) noise data can be generated using the estimated $\phi_{\gamma}$. If the estimation is accurate enough, the eigenvalues for simulated AR(1) noise data using the estimated $\phi_{\gamma}$ can be fitted by an exponent function $\lambda_{1}(k)=a^{\prime} \mathrm{e}^{-b k}$, which has the same $b$ as the real dataset. The domain of definition of $\lambda_{1}(k)$ is $k=1,2, \ldots, M$. In order to make comparison easy, $\lambda_{0}(k)$ is also extended to $k=1,2, \ldots, M$ following the exponent function $\lambda_{0}(k)=a \mathrm{e}^{-b k}$.

Real data contain both signal and noise and the corresponding simulated pure AR(1) noise data contain only noise, and each time series is normalized by its variance. Therefore, the eigenvalues of the covariance matrices form the pattern

$M=\sum_{k=1}^{M} a^{\prime} e^{-b k}>\sum_{k=1}^{M} a e^{-b k}$

Using inequality (a1), it is proved easily that the inequality $a<a^{\prime}$ can hold. Thus, we can obtain the inequality $\lambda_{0}(i)=a \mathrm{e}^{-b i}<$ $a^{\prime} \mathrm{e}^{-b i}=\lambda_{1}(i), \forall i \in[p+1, M]$. This means that the tail eigenvalues of covariance matrix for the real data are always smaller than the corresponding eigenvalues for simulated pure $\operatorname{AR}(1)$ noise. The shift $\Delta$ can be obtained by the mean of differences between tail eigenvalues of real data and those of simulated $\mathrm{AR}(1)$ noise data. 


\section{References}

Bandettini, P.A., Birn, R.M., Kelley, D., Saad, Z.S., 2002. Dynamics nonlinearities in BOLD contrast: neuronal or hemodynamic. Internation Congress Series 1235, 73-85.

Bartels, A., Zeki, S., 2005. Brain dynamics during natural viewing condition-A new guide for mapping connectivity in vivo. NeuroImage 24, 339-349.

Birn, R.M., Saad, Z.S., Bandettini, P.A., 2001. Spatial Heterogenenity of the Nonlinear Dynamics in the FMRI BOLD Response. NeuroImage 14, $817-826$.

Bianciardi, M., Sirabella, P., Hagberg, G.E., Giuliani, A., Zbilut, J.P., Colosimo, A., 2007. Model-free analysis of brain fMRI data by recurrence quantification. NeuroImage 37, 489-503.

Breakspear, M., Brammer, M., Robinson, P.A., 2003. Construction of multivariate surrogate sets from nonlinear data using the wavelet transform. Physica D 182, 1-22.

Bullmore, E., Fadili, J., Maxim, V., Sendur, L., Whitcher, B., Suckling, J., Brammer, M., Breakspear, M., 2004. Wavelets and functional resonance imaging of the human brain. NeuroImage 23, S234-S249.

Burden, R.L., Faires, J.D., 2001. Numerical analysis. Higher Education Press and Thomson Learning, Inc., Beijing.

Buxton, R.B., Wong, E.C., Frank, L.R., 1998. Dynamics of blood flow and oxygenation changes during brain activation: the balloon model. Magn. Reson. Med. 39 (6), 855-864.

Cellucci, C.J., Albano, A.M., Rapp, P.E., 2003. Comparative study of embedding method. Phys. Rev. E 67 066210-1-13.

Cordes, D., Nandy, R.R., 2006. Estimation of the intrinsic dimensionality of fMRI data. NeuroImage 29, 145-154.

Cordes, D., Haughton, V.M., Arfanakis, K., Carew, J.D., Turski, P.A., Moritz, C.H., Quigley, M.A., Meyerand, M.E., 2001. Frequencies Contributing to Functional Connectivity in the Cerebral Cortex in "Resting-state" Data. AJNR Am. J. Neuroradiol. 22, 1326-1333.

Deshpande, G., LaConte, S., Peltier, S., Hu, X., 2005. Spatial Embedding of fMRI for Investigating Local Coupling in Human Brain. Medical Imaging 2005: Physiology, Function, and Structure from Medical Images, Proceeding of SPIE, Vol. 5746, pp. 119-125. SPIE, Bellingham, WA.

Deshpande, G., LaConte, S., Peltier, S., Hu, X., 2006. Tissue Specificity of Nonlinear Dynamics in Baseline fMRI. Magn. Reson. Med. 55, $626-632$.

Dhamala, M., Pagnoni, G., Wiesenfeld, K., Berns, G.S., 2002. Measurement of brain activity complexity for vary mental loads. Phys. Rev. E 65, 041917-1-7.

Freeman, W.J., 2000. Mescopic neurodynamics: From neuron to brain. J. Physiol. (Paris) 94, 303-322.

Friman, O., Westin, C.F., 2005. Resampling fMRI time series. NeuroImage $25,859-867$.

Friston, K.J., Mechelli, A., Turner, R., Price, C.J., 2000. Nonlinear Responses in fMRI: The Balloon Model, Volterra Kernels, and Other Hemodynamics. NeuroImage 12, 466-477.

Galka, A., 2000. Topics in nonlinear time series analysis: with implications for EEG analysis. World Scientific, Singppore.

Gautanma, T., Mandic, D.P., Hulle, M.M.V., 2003. Signal nonlinearity in fMRI: A comparison between BOLD and MION. IEEE Trans. Med. Imag. 22 (5), 636-644.

Glass, L., Mackey, M.C., 1988. From clocks to chaos: the rhythms of life. Princeton University Press, Princeton, New Jersey.

Gusnard, D.A., Raichle, M.E., 2001. Searching for a baseline: functional imaging and the resting human brain. Nat. Rev., Neurosci. 2, 685-694.

Hampson, M., Peterson, B.S., Skudlarski, P., Gatenby, J.C., Gore, J.C., 2002. Detection of Functional Connectivity Using Temporal Correlations in MR Images. Hum. Brain Mapp. 15, 247-262.

Harrison, L., Penny, W.D., Friston, K., 2003. Multivariate autoregressive modeling of fMRI time series. NeuroImage 19, 1477-1491.

Hegger, R., Kantz, H., 1999. Practical implementation of nonlinear time series methods: The TISEAN package. Chaos 9, 413-435.

Huettel, S.A., 2004. Non-linearities in blood-oxygenation-level dependent
(BOLD) response measured by functional magnetic resonance imaging (fMRI). Proceeding of the 26th Annual International Conference of the IEEE EMBS, San Francisco, CA, pp. 4413-4416.

Kantz, H., Schreiber, T., 1997. Nonlinear time series analysis. Cambridge University Press, Cambridge.

LaConte, S., Peltier, S., Kadah, Y., Ngan, S.C., Deshpande, G., Hu, X., 2004 Detecting nonlinear dynamics of functional connectivity. Medical Imaging 2004: Physiology, Function, and Structure from Medical Images, Proceeding of SPIE, Vol. 5369, pp. 227-237. SPIE, Bellingham, WA.

Lahaye, P.J., Poline, J.B., Flandin, G., Dodel, S., Garnero, L., 2003. Functional connectivity: studying nonlinear, delayed interactions between BOLD signals. NeuroImage 20, 962-974.

Laird, A.R., Rogers, B.P., Meyerand, M.E., 2002. Investigating the Nonlinearity of fMRI Activation Data. Proceeding of the Second Joint EMBS/BMES Conference. IEEE, Houston, TX, pp. 11-12.

Lee, U., Kim, S., 2006. Classification of epilepsy types through global network analysis of scalp electroencephalograms. Phys. Rev. E 73, 041920-1-9.

Lee, Y.J., Zhu, Y.S., Xu, Y.H., Shen, M.F., Zhang, H.X., Thakor, N.V., 2001. Detection of non-linearity in the EEG of schizophrenic patients. Clin. Neurophysiol. 112, 1288-1294.

Lehnertz, K., Elger, C.E., 1998. Can epileptic seizures be predicted? Evidence from nonlinear time series analysis of brain electrical activity. Phys. Rev. Lett. 80 (22), 5019-5022.

Lowe, M.J., Mock, B.J., Sorenson, J.A., 1998. Functional Connectivity in Single and Mutislice Echoplanar Imageing Using Resting-State Fluctuation. NeuroImage 7, 119-132.

Luca, D.M., Beckmann, C.F., Stefano, D.N., Matthews, P.M., Smith, S.M., 2006. fMRI resting state networks define distinct modes of long-distance interactions in the human brain. NeuroImage 29, 1359-1367.

Maxim, V., Sendur, L., Fadili, J., Suxkling, J., Gould, R., Howard, R., Bullmore, E., 2005. Fractional Gaussian noise, functional MRI and Alzheimer's diease. NeuroImage 25, 141-158.

McIntosh, A.R., Chan, W.K., Protzner, A.B., 2004. Spatiotemporal analysis of event-related fMRI data using partial least squares. NeuroImage 23, 764-775.

Müller, M., Baier, G., 2005. Detection and characterization of changes of the correlation structure in multivariate time series. Phys. Rev. E 71 046116-1-16.

Pezard, L., Martinerie, J., Müller-Gerking, J., Varela, F.J., Renault, B., 1996. Entropy quantification of human brain spatio-temporal dynamics. Physica D 96, 344-354.

Pfeuffer, J., McCullough, J.C., Moortele, V.P.F., Ugurbil, K., Hu, X., 2003. Spatial dependence of the nonlinear BOLD response at short stimulus duration. NeuroImage 18, 990-1000.

Perc, M., 2005. Nonlinear time series analysis of the human electrocardiogram. Eur. J. Phys. 26, 757-768.

Reidl, J., Strrke, J., Omer, D.B., Grinvald, A., Spors, H., 2007. Independent component analysis of high-resolution imaging data identifies distinct functional domains. NeuroImaging 34, 94-108.

Raichle, M.E., 2006. The brain's dark energy. Science 314, 1249-1250.

Ricard, V.S., Jordi, B., 1995. Measuing Chaos from Spatial Information. J. Theor. Biol. 175, 139-147.

Raab, C., Kurths, J., 2001. Estimation of large-scale dimension densities. Phys. Rev. E 64, 016216-1-5.

Raab, C., Wessel, N., Schirdewan, A., Kurths, J., 2006. Large-scale dimension densities for heart rate variability analysis. Phys. Rev. E 73, 041907-1-7.

Schreiber, T., Schmitz, A., 2000. Surrogated time series. Physica D 142, $346-382$.

Sendur, L., Suckling, J., Whitcher, B., Bullmore, E., 2007. Resampling methods for improved wavelet-based multiple hypothesis testing of parametric maps in functional MRI. NeuroImage 37, 1186-1194.

Small, M., 2005. Applied nonlinear time series analysis: applications in physics, physiology and finance. World Scientific Publishing Co. Pet. Ltd., Singapore.

Stam, C.J., 2005. Nonlinear dynamical analysis of EEG and MEG: Review of an emerging field. Clin. Neurophysiol. 116, 2266-2301. 
Stam, C.J., Jelles, B., Achtereekte, H.A.M., Rombouts, S.A.R.B., Slaets, J.P.J., Keunen, R.W.M., 1995. Investigation of EEG non-linearity in dementia and Parkinson's disease. Electroencephalogr. Neurophysiol. 95, 309-317.

Thirion, B., Dodel, S., Poline, J.B., 2006. Detection of signal synchronizations in resting-state fMRI data. NeuroImage 29, 321-327.

Uhl, C., Kruggel, F., Opitz, B., Cramon, D.Y., 1998. A New Concept for EEG/MEG Signal Analysis: Detection of Interacting Spatial Modes. Hum. Brain Mapp. 6, 137-149.

Vazquez, A.L., Noll, D.C., 1998. Nonlinear Aspects of the BOLD Response in Functional MRI. NeuroImage 7, 108-118.

Wager, T.D., Vazquez, A., Hernandez, L., Noll, D.C., 2005. Accounting for nonlinear BOLD effects in fMRI: parameter estimates and a model for prediction in rapid event-related studies. NeuroImage 25, 206-218.

Wicker, B., Ruby, P., Royet, J.P., Fonlupt, P., 2003. A relation between rest and self in the brain? Brain Res. Rev. 43, 224-230.

Worsley, K.J., Chen, J.I., Lerch, J., Evans, A.E., 2005. Comparing functional connectivity via thresholding correlations and singular value decomposition. Phil. Trans. R. Soc. B 360, 913-920.

Zuendorf, G., Kerrouche, N., Herholz, K., Baron, J.C., 2003. Efficient Principal Component Analysis for Multivariate 3D Voxel-Based Mapping of Brain Functional Imaging Data Sets as Applied to FDGPET and Normal Aging. Hum. Brain Mapp. 18, 13-21. 